\title{
Switching properties of ferromagnetic nanoparticles driven by a circularly polarized magnetic field
}

\author{
T V Lyutyy ${ }^{1}$, A Yu Polyakov ${ }^{1}$, A V Rot-Serov ${ }^{1}$ and C Binns ${ }^{2}$ \\ ${ }^{1}$ Sumy State University, 2 Rimsky-Korsakov Street, 40007 Sumy, Ukraine \\ ${ }^{2}$ University of Leicester, Leicester LE1 7RH, UK \\ E-mail: lyutyy@oeph.sumdu.edu.ua
}

Received 8 April 2009, in final form 24 July 2009

Published 8 September 2009

Online at stacks.iop.org/JPhysCM/21/396002

\begin{abstract}
We present a comprehensive study of the magnetization switching of a uniaxial nanoparticle driven by a circularly polarized magnetic field rotated in the plane perpendicular to the easy axis. The conditions for the existence of the uniform and non-uniform precessions of the nanoparticle magnetic moment are derived. In addition, the differences between switchings via uniform and non-uniform precession are determined, and the essential role of field polarization is demonstrated. The dependence of the switching time on the field amplitude and frequency are calculated numerically. We show that a permanent magnetic field can reduce the amplitude and frequency of the switching rotating field, and that the combined action of these fields is characterized by an extremely strong dependence of the switching time on the field parameters. We also demonstrate that the transition process caused by an external magnetic field pulse can decrease the switching amplitude in comparison with the value predicted from analysis of the stability criterion. We discuss the advantages of switching the magnetization by means of the action of a rotating field over the magnetization switching using a steady field applied perpendicular to the easy axis.
\end{abstract}

(Some figures in this article are in colour only in the electronic version)

\section{Introduction}

Magnetic fine particles and their ordered ensembles are receiving intense attention due to a fundamental interest in nanomagnetism and also for practical applications. The scientific interest arises from novel behavior associated with magnetism at the nanoscale including giant magnetoresistance [1, 2], quantum tunneling of magnetization [3, 4] and finite-size and surface effects $[6,5]$. For applications their importance arises from the potential of nanoparticle arrays in producing new electronic devices such as ultra-high density storage medias [7-9] and gauges [10].

The magnetization is a fundamental property of a ferromagnetic particle that depends on each spin of the crystal lattice and is defined by a mean value of the spin. When the exchange coupling between such spins is strong enough, the magnetization of each nanoparticle can be characterized by a magnetic moment vector $\vec{\mu}$ with a constant magnitude. In this case the dynamics of $\vec{\mu}$ is described by the model of coherent rotation [11, 12]. For particles with a uniaxial magnetic anisotropy the magnetic moment has two equilibrium states separated by a potential barrier. These two states can be used to represent a binary bit and the problem of controlled switching between them is central to recording information. In this connection, two parameters are important. Firstly there is the stability of $\vec{\mu}$ relative to thermal fluctuations that determines the reliability of the storage devices. For non-interacting particles this was studied in [13]. The case of particles interacting by dipolar interactions was studied analytically (see, e.g. in [14-17]), numerically (see, e.g. in [18-21]), and experimentally (see, e.g. in [22-24]). Secondly, there is the controlled switching of $\vec{\mu}$. For the best device performance the switching between two equilibrium states must be fast and have low power consumption. The nanoparticle magnetization reversal process is driven by the external magnetic field and its character depends on external field parameters 
such as direction, magnitude and duration of the field action.

The simplest type of external field for switching the nanoparticle magnetic moment is a permanent field $\vec{H}$ applied along the easy axis. The exact description of the magnetization dynamics under such a field action was given in [25]. Later it was shown that transitions between equilibrium states can occur for other types of fields. For instance, in [26-28] the switching under external field $\vec{H}_{\perp}$ pulses applied perpendicular to the easy axis of the particle was considered. In particular it was shown that in this case the time needed for magnetization reversal is much smaller than for a longitudinal field $\vec{H}$. The combined action of a permanent field, $\vec{H}$, applied along the easy axis and a field, $\vec{h}(t)$, which is circularly polarized in the plane perpendicular to the easy axis, was studied in [29]. Here the value of the critical field $\vec{H}_{\text {cr }}$ for switching is appreciably smaller in comparison with applying only the field $\vec{H}$. This was confirmed by experiments on magnetization reversal of nanoparticles in the presence of a radio-frequency rotating field pulse [30].

The idea of switching nanoparticle magnetization by a circularly polarized field $\vec{h}(t)$ was further developed in [31-33]. Based on the stability criteria, it was shown analytically [32] that such switching can occur only under the action of the field $\vec{h}(t)$ if the precession of magnetization becomes unstable for $|\vec{h}(t)|=h_{\mathrm{cr}}$. However, loss of stability in some cases leads to transitions between uniform and non-uniform precession of the magnetization [34] without switching. Non-uniform precession consists of periodic oscillations of the precession angle, so-called nutations, and their presence indicates a fundamental change of the magnetization dynamics.

An original approach was proposed in [35] in which the main feature is the dependence of the external field shape on the current position of the nanoparticle magnetic moment. Within this framework the theoretical limits for the minimal amplitude of the switching field, and minimal switching time were obtained. It is difficult to realize this idea in practice however due to the complicated shape of the external field required.

It is important therefore to focus on switching parameters under the action of external fields that are straightforward to produce. As was shown in the aforementioned work and from our own investigations, using a circularly polarized field $\vec{h}(t)$ is very promising for the magnetization reversal process. Due to the presence of a non-uniform precession mode of $\vec{\mu}$, the magnetization switching is too complicated for an analytical solution and, as a first approach, it is appropriate to use computer modeling. The numerical results for the optimal characteristics of switching fields and switching times are presented here. The paper is organized as follows. In section 2 the model is considered and basic equations are given. Based on the stability criterion, the critical value for the switching field amplitude is derived analytically. In section 3 the results for comprehensive numerical studies of switching nanoparticle magnetization via a rotating magnetic field are performed. In particular, uniform and non-uniform modes of $\vec{\mu}$ precession are delimited and parameters of switching, such as minimal

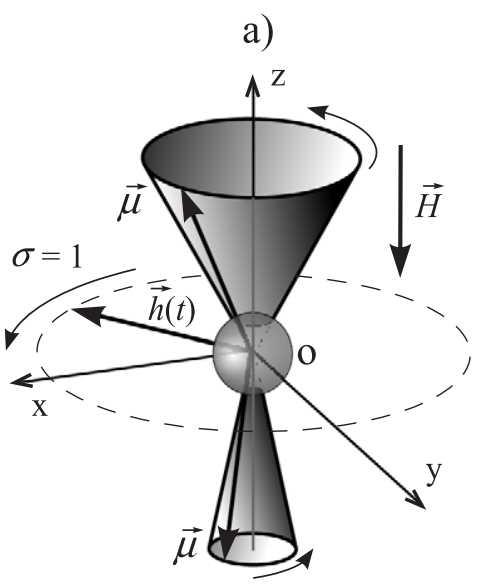

b)

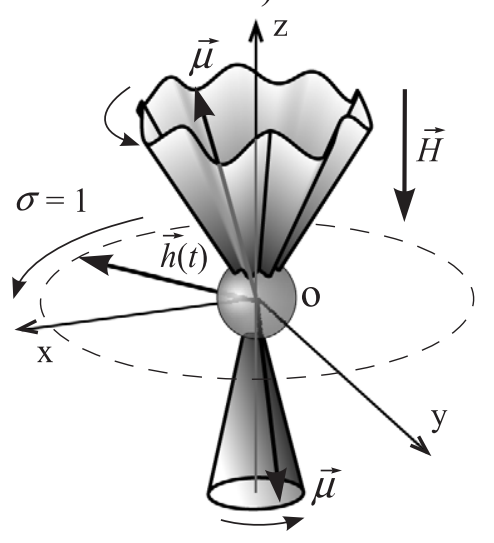

Figure 1. Schematic representation of a ferromagnetic nanoparticle with magnetic moment $\vec{\mu}$, the applied fields, and the coordinate system used. (a) Uniform precession; (b) non-uniform precession of $\vec{\mu}$.

amplitude and switching time are derived. In addition the effect of a pulsed external field is investigated. We summarize our finding in section 4.

\section{Description of the model}

The object of investigation is a uniaxial ferromagnetic nanoparticle that is characterized by the anisotropy field $H_{\mathrm{a}}$ and the magnetic moment $\vec{\mu}=\vec{\mu}(t)$ of fixed magnitude $|\vec{\mu}|=\mu$. Such a particle is under the influence of a permanent magnetic field $\vec{H}$, applied along the easy axis of magnetization (the $z$ axis), and a circularly polarized magnetic field $\vec{h}(t)$, that is applied in the $x y$ plane, i.e., $\vec{H}=H \vec{e}_{z}$ and

$$
\vec{h}(t)=h \cos (\omega t) \vec{e}_{x}+\sigma h \sin (\omega t) \vec{e}_{y} .
$$

Here $\vec{e}_{x}, \vec{e}_{y}$, and $\vec{e}_{z}$ are the unit vectors along the corresponding axes of the Cartesian coordinate system $x y z$, $h=|\vec{h}(t)|, \quad \omega$ is the frequency of $\vec{h}(t)$, and $\sigma=$ -1 or +1 correspond to clockwise (right polarization) or counterclockwise (left polarization) rotation of $\vec{h}(t)$, respectively (see figure 1). The magnetic energy of a 
nanoparticle in such a field can be written in the form

$$
W=-\frac{H_{\mathrm{a}}}{2 \mu} \mu_{z}^{2}-H \mu_{z}-\vec{\mu} \cdot \vec{h}(t),
$$

where $\mu_{z}=\vec{\mu} \cdot \vec{e}_{z}$ is the $z$ component of $\vec{\mu}$, and the dot denotes the scalar product.

We designate the projection of $\vec{\mu}$ on the $z$ axis as positive for the 'up state' and negative for the 'down state'. In the absence of thermal fluctuation, the dynamics of the nanoparticle magnetic moment is described by the LandauLifshitz equation [36]

$$
\dot{\vec{\mu}}=-\gamma \vec{\mu} \times \vec{H}_{\mathrm{eff}}-\frac{\lambda \gamma}{\mu} \vec{\mu} \times\left(\vec{\mu} \times \vec{H}_{\mathrm{eff}}\right) .
$$

Here $\gamma \approx 1.7609 \times 10^{11} \mathrm{rad} \mathrm{s}^{-1} \mathrm{~T}^{-1}$ is the gyromagnetic ratio, $\lambda(>0)$ is the dimensionless damping parameter, the cross denotes the vector product, the dot over $\vec{\mu}$ denotes the first derivative with respect to time, and

$$
\vec{H}_{\text {eff }}=-\frac{\partial W}{\partial \vec{\mu}}=\vec{h}(t)+\left(H_{\mathrm{a}} \frac{\mu_{z}}{\mu}+H\right) \vec{e}_{z}
$$

is the effective magnetic field acting on $\vec{\mu}$. Due to the spherical symmetry of the problem, and taking into account equations (1), (2), and (4), the Landau-Lifshitz equation (3) can be represented as a system of differential equations for angular coordinates of $\vec{\mu}$ :

$$
\begin{gathered}
\dot{\theta}=\tilde{h} \sin \phi-\lambda(\sin \theta \cos \theta-\tilde{H} \cos \theta-\tilde{h} \cos \theta \cos \phi), \\
\dot{\varphi}=\cos \theta+\tilde{H}-\cot \theta \cos \phi-\frac{\lambda}{\sin \theta} \tilde{h} \sin \phi,
\end{gathered}
$$

where $\phi=\varphi-\sigma \tilde{\omega} t$, with $\theta$ and $\varphi$ being the polar and azimuthal angles of the vector $\vec{\mu}$ respectively, $\tilde{H}=H / H_{\mathrm{a}}$, $\tilde{h}=h / H_{\mathrm{a}}, \tilde{\omega}=\omega / \omega_{\mathrm{r}}$, and $\omega_{\mathrm{r}}$ is a resonance frequency $\left(\omega_{\mathrm{r}}=\gamma H_{\mathrm{a}}\right)$. For the uniform mode, equations (5) can be transformed [32] into the algebraic equation

$$
\tilde{h}^{2}=\frac{1-\cos ^{2} \theta}{\cos ^{2} \theta}\left[(\cos \theta+\tilde{H}-\sigma \kappa)^{2}+(\lambda \kappa \cos \theta)^{2}\right],
$$

where $\kappa=\tilde{\omega} /\left(1+\lambda^{2}\right)$. In the first-order approximation, the stability of the steady-state precession of the nanoparticle magnetic moment is defined by the stability of the stationary solution of the linearized system of equations corresponding to the system (5) with respect to small perturbations. From calculations performed in [32] it follows that a criterion of the asymptotic stability of the forced precession is given by the equation

$$
\beta=\lambda\left(\rho_{1}+\rho_{4}\right)-\operatorname{Re} \sqrt{\lambda^{2}\left(\rho_{4}-\rho_{1}\right)^{2}-4 \rho_{2} \rho_{3}}
$$

with

$$
\begin{gathered}
\rho_{1}=\cos ^{2} \theta+\frac{1}{\cos \theta}\left[\tilde{H}-\sigma \kappa\left(1-\cos ^{2} \theta\right)\right], \\
\rho_{2}=1+\frac{1}{\cos \theta}\left[\tilde{H}-\sigma \kappa\left(1+\lambda^{2} \cos ^{2} \theta\right)\right], \\
\rho_{3}=\cos ^{2} \theta+\frac{1}{\cos \theta}\left[\tilde{H}-\sigma \kappa\left(1+\lambda^{2} \cos ^{2} \theta\right)\right], \\
\rho_{4}=1+\frac{1}{\cos \theta}\left[\tilde{H}-\sigma \kappa\left(1-\cos ^{2} \theta\right)\right] .
\end{gathered}
$$

If the condition $\beta \geqslant 0$ holds, the uniform mode for the precession of $\vec{\mu}$ is realized (see figure 1(a)). The motion within such a mode is characterized by a fixed angle for the precession cone. In accordance with terms used in [34], we call this type of motion the $\mathbf{P}$-mode. In the opposite case $(\beta<0)$ two different situations are possible. One is the nanoparticle magnetization switching as predicted in [32]. This effect can be very interesting for the design of magnetic recording devices. The other is a non-uniform mode of the $\vec{\mu}$ precession that is characterized by periodic changes of the angle of the precession cone (see figure 1(b)). In accordance with terms used in [34], we call this type of motion the Q-mode. It is important to point out, that switching or non-uniform mode generation occurs only when the directions of the external field rotation and natural precession of $\vec{\mu}$ coincide.

\section{Numerical study}

\subsection{Delimitation uniform and non-uniform modes}

From a practical point of view it is necessary to study a whole range of parameters for the switching fields in order to select an optimal one. The starting point of this problem is the delimitation of non-uniform and uniform precession of the nanoparticle's magnetic moment. Let us consider the algebraical equation $\beta=0$, where $\beta$ is given by equation (7). This equation can have up to four real roots, and the largest one determines the maximal value of the precession angle for the uniform mode. In turn, using equation (6), one can find a critical amplitude of a circularly polarized field $\tilde{h}_{\text {cr }}$, corresponding to the given field frequency $\tilde{\omega}$. The significance of $\tilde{h}_{\text {cr }}$ is the following: when the field amplitude $\tilde{h}$, increasing in a quasistatic fashion, reaches the critical value $\tilde{h}_{\mathrm{cr}}$, the magnetization dynamics changes mode.

Figure 2 shows the results of the numerical solution of equations (5) and algebraic equation $\beta=0$. Here and below the value of the dissipation parameter is $\lambda=0.2$. The comparison of these results enables us to evaluate the validity of an analytical approach based on the stability criteria developed in [32]. It is also very useful to get a full conception of the types of motion and transitions between them. The solid black line in figure 2(a) shows, for sufficiently small field frequencies $\tilde{\omega}$, the boundary between the up (region 1) and down (region 4 ) states of the magnetic moment $\vec{\mu}$. A transition between region 1 and 4 when $\tilde{h}=\tilde{h}_{\mathrm{cr}}$ is a transition between two $\mathbf{P}$-modes, located in up and down states of the nanoparticle magnetic moment. So, in this case $\tilde{h}_{\mathrm{cr}}$ is a minimal amplitude of the switching field, corresponding to the given frequency $\tilde{\omega}$.

When the field frequency approaches the resonance value, $\tilde{h}_{\mathrm{cr}}$ denotes the start of the non-uniform or Q-mode. In figure 2(a) such a transition is depicted by circular markers, and the $\mathbf{Q}$-mode is represented by region 3. Region 2, or socalled intermediate $\mathbf{P}$-mode, deserves special attention. Here the line $\tilde{h}_{\text {cr }}=\tilde{h}_{\text {cr }}(\tilde{\omega})$ represents a jump between two $\mathbf{P}$-modes located in the up state of the magnetic moment and differing by the angle of the precession cone. Transitions between regions 1 and 2 are irreversible and region 2 disappears in the case of $\tilde{h}$ decreasing in a quasistatic fashion. The numerical solution 
a)

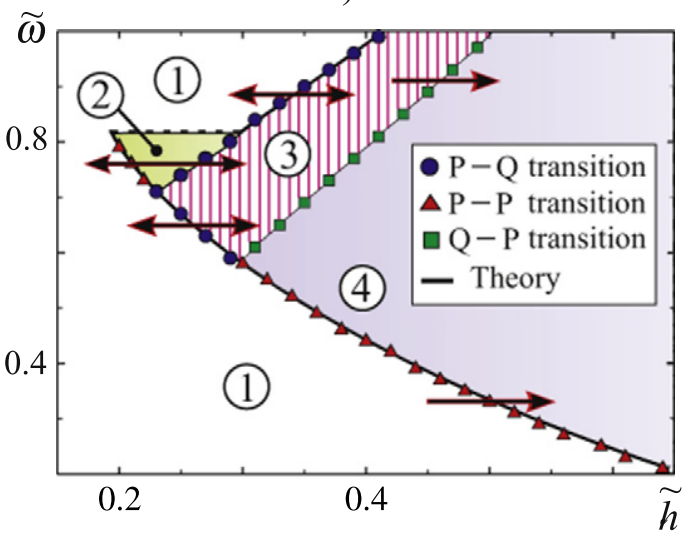

b)

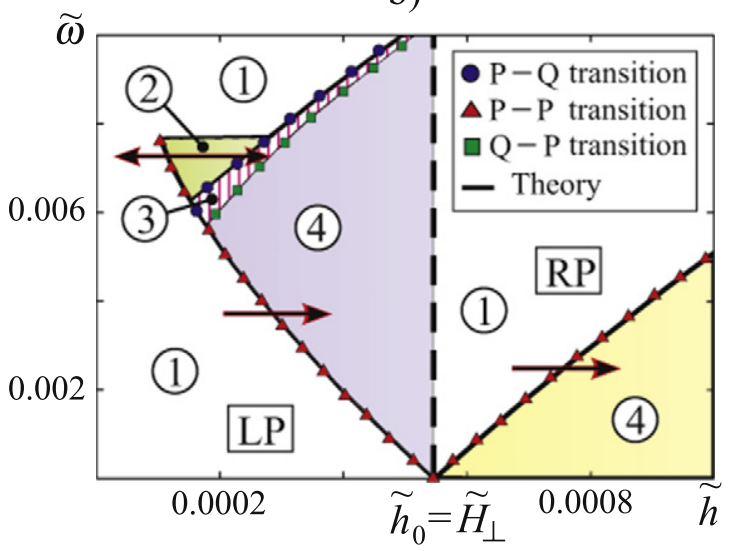

Figure 2. Precession modes of the $\vec{\mu}$ that are realized under increasing $\tilde{h}$ in a quasistatic fashion. (a) The case of $\tilde{H}=0$ for right polarization only; (b) the case of $\tilde{H}=-0.99$ for right (RP) and left (LP) polarization of the rotating field: (1) the region, corresponding to the $\mathbf{P}$-mode in the up state of $\vec{\mu},(2)$ the region, corresponding to the intermediate $\mathbf{P}$-mode in the up state of $\vec{\mu}$, (3) the region, corresponding to the $\mathbf{Q}$-mode in the up state of $\vec{\mu}$, (4) the region, corresponding to the $\mathbf{P}$-mode in the down state of $\vec{\mu}$.

of equations (5) for the field amplitudes with $\tilde{h}>\tilde{h}_{\text {cr }}$ shows that the Q-mode can become unstable too and $\vec{\mu}$ is switched into the $\mathbf{P}$-mode located in the down state (see figure 2(a)). The transition between regions 3 and 4 is depicted by square markers. So, switching through the non-uniform mode is observed.

The main disadvantage of switching magnetization via a rotating field is the high frequency $(\tilde{\omega} \sim 1)$ and large amplitude $(\tilde{h} \sim 0.1)$ required. So, it is interesting to investigate the parameters when the circularly polarized field $\tilde{h}(t)$ is applied in combination with a static field $\tilde{H}$, antiparallel to $z$ axis. The results of the simulations for $\tilde{H}=-0.99$ are shown in figure 2(b). In general, the nanoparticle magnetization demonstrates the same dynamics, but the amplitude and frequency of the switching field are significantly reduced in comparison with the previous case, where $\tilde{H}=0$. However, there are three important features. First, the switching of magnetic moment is possible for left polarization $(\sigma=+1)$ of the field and for right polarization $(\sigma=-1)$ as well. As is observed in figure 2(b) however, a smaller amplitude is required for switching the magnetization in the case of left polarization. Second, the region for the $\mathbf{Q}$-mode is thinner than in the case of $\tilde{H}=0$ (see figure 2). As shown below this last observation can set conditions for a different reaction to the turning on of an external field. The curves $\tilde{h}_{\mathrm{cr}}(\tilde{\omega})$ for the left and right polarizations of the external field coincide at $\tilde{h}_{0}$ when $\tilde{\omega}=0$. So, $\tilde{h}_{0}$ corresponds to the value of the permanent field $\tilde{H}_{\perp}=\left|\vec{H}_{\perp}\right| / H_{\mathrm{a}}$ perpendicular to the easy axis that switches $\vec{\mu}$ as well. The final feature of the switching process caused by the combined action of $\vec{h}(t)$ and $\vec{H}$ that we would like to highlight concerns the direction of the $\vec{\mu}$ rotation. The circularly polarized field can switch the nanoparticle magnetic moment only if the field rotation coincides with natural precession of the $\vec{\mu}$. At the same time, application of the field $\vec{H}$ leads to a precession of $\vec{\mu}$ in the opposite direction. As far as the condition $\tilde{H} \gg \tilde{h}$ holds, $\vec{\mu}$ rotates in a clockwise direction during the switching process.

The theoretical analysis, represented above, was based on the stability under small perturbations. Then in the numerical simulation we change the field amplitude in a quasistatic fashion. So, such an approach is suitable only if the field amplitude grows very slowly. But in a real write process, when the external field is turned on, its amplitude grows very fast. During such external action the magnetic moment evolves from an initial equilibrium state, that is characterized by a polar angle $\theta \rightarrow 0$ to the new equilibrium state that is characterized by a new angle $\theta>0$. It is accompanied with the oscillations of the angle $\theta$ similar to nutations, but with a damped amplitude. Such changes are also called transition processes. Since the amplitude of the oscillation can be considerable, it is expected that switching of the nanoparticle magnetization can occur for external field amplitudes different to $\tilde{h}_{\mathrm{cr}}$.

To confirm this, we simulated the magnetic moment response to the action of the field jump from zero to the defined value of amplitude. In figure 3(a) the dependence of $\tilde{h}_{\mathrm{cr}}(\tilde{\omega})$ and the curve $\tilde{h}_{\min }(\tilde{\omega})$, calculated numerically for the switch on of the external field, are depicted. The parameter $\tilde{h}_{\text {min }}$ represents the minimal field amplitude for which the magnetization reversal is possible in the case of the field turning on. As we can see, when the $\mathbf{Q}$-mode is absent, the switching field amplitude $\tilde{h}_{\text {min }}$ is smaller than the theoretically predicted value of $\tilde{h}_{\mathrm{cr}}$. In the opposite case however, when the $\mathbf{Q}$-mode can be generated, the magnetization switching is possible only for field amplitudes corresponding to the transition between regions 3 and 4 (see figure 2). So, the actual switching field amplitude is larger than $\tilde{h}_{\mathrm{cr}}$. These results show again the importance of taking into account the non-uniform mode in order to choose optimal parameters for a switching field. The results for similar simulations when $\tilde{H}=-0.99$, are shown in figure 3(b). The main difference from the case $\tilde{H}=0$ is that due to the small thickness of the $\mathbf{Q}$-mode region, the transition processes can overcome this region when the field frequencies are large enough. So, the switching of the magnetization occurs similarly to the case for smaller field frequencies where the $\mathbf{Q}$-mode is absent. 
a)

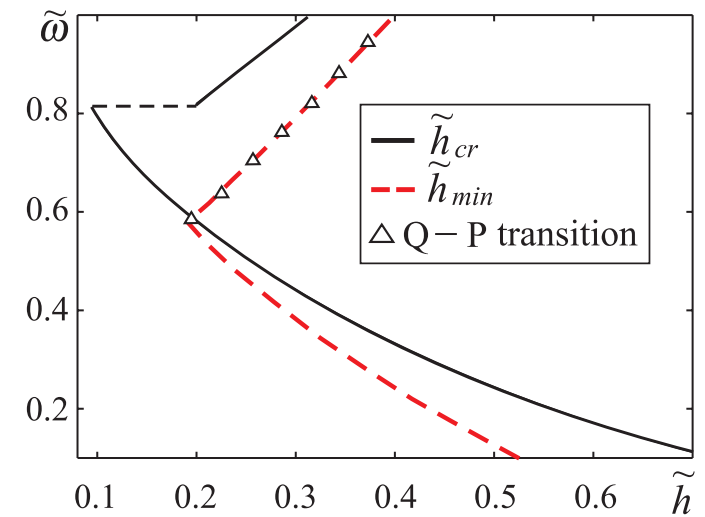

b)

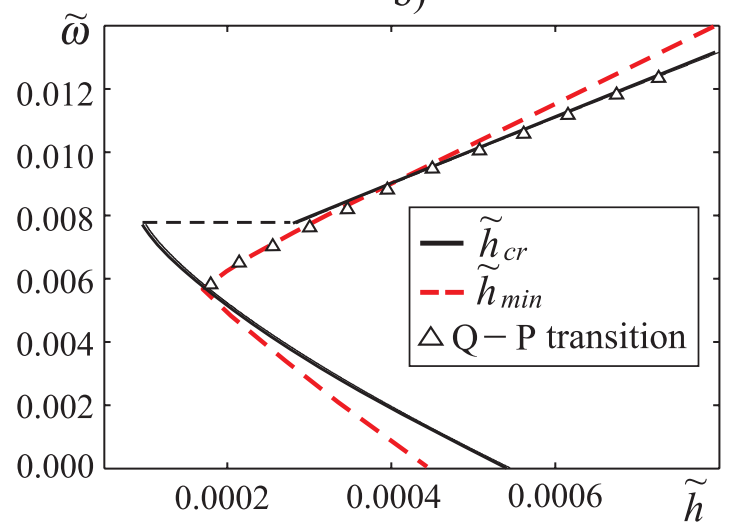

Figure 3. Minimal switching field's amplitudes. (a) The case of $\tilde{H}=0$; (b) the case of $\tilde{H}=-0.99$.

\subsection{Switching time}

Switching time is a parameter that ultimately determines the performance of recording media devices. When choosing the amplitude and frequency for the switching field it is necessary to take into account how fast the magnetization reversal of a nanoparticle will occur. The formal definition of switching time was given in [35]

$$
T_{\mathrm{sw}}=\int_{0}^{\theta_{0}} \frac{\mathrm{d} \theta}{\dot{\theta}} .
$$

Depending on the value of the constant $\theta_{0}$, there are several interpretations for switching time. In [35] it was assumed that $\theta_{0}=\pi$ in which case $T_{\mathrm{sw}}$ is the exact time required to reach the down state. Such an assumption leads to a strong dependence of $T_{\mathrm{sw}}$ on the precision of the calculations of the angle $\theta$. In addition, the ground state of $\vec{\mu}$ under the action of a rotating field does not coincide with the easy axis (see figure 1). In [30], $T_{\mathrm{sw}}$ is the time required for the angle $\theta$ to first reach $\pi / 2$, so $\theta_{0}=\pi / 2$. Such an approach neglects the possibility of $\vec{\mu}$ returning back, which is especially important for the non-uniform mode of precession. In our investigations we assume, that $T_{\mathrm{sw}}$ is the time before the last crossing of the $x y$ plane by the vector $\vec{\mu}$ during the reversal process. Due to the difficulty of direct integration of equation (9), we find $T_{\mathrm{sw}}$ numerically. a)

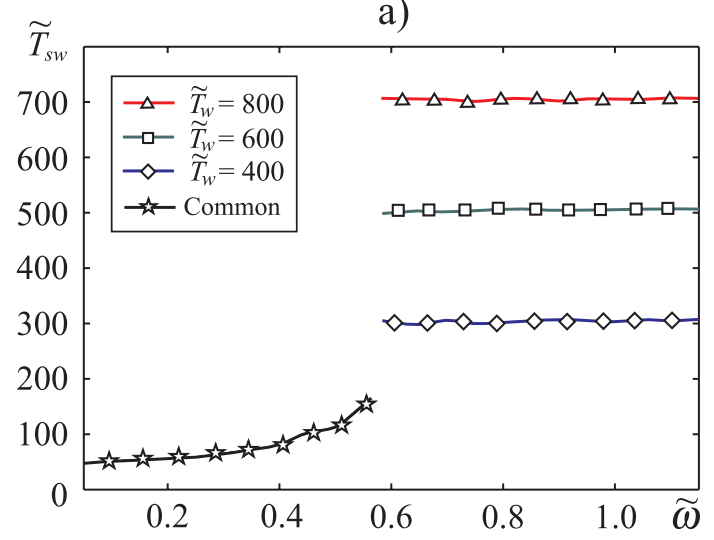

b)

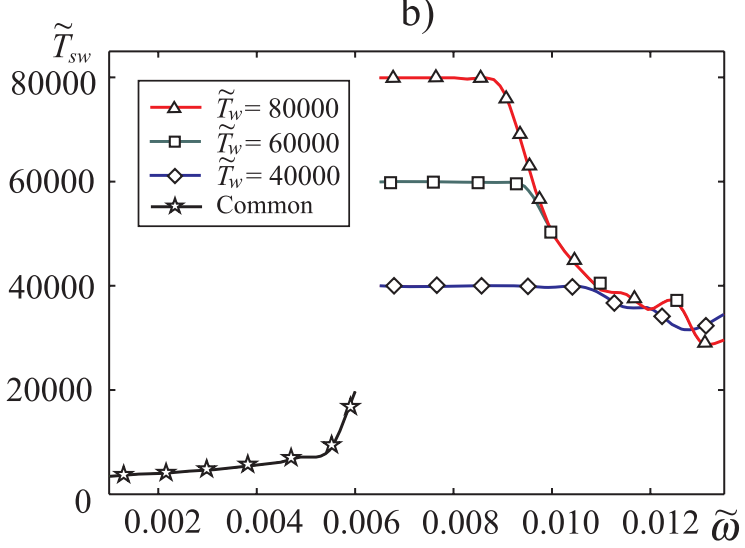

Figure 4. Switching time, calculated in the resonant period, corresponding to the $\tilde{h}_{\min }$ for different waiting times. (a) The case of $\tilde{H}=0$; (b) the case of $\tilde{H}=-0.99$.

It is clear that the switching time increases with decreasing $\tilde{h}$. Since the minimal amplitude of switching field decreases with frequency in the absence of the non-uniform mode (see figure 3), the values of switching time $\tilde{T}_{\mathrm{sw}}$ corresponding to the minimal value of the rotating field amplitude $\tilde{h}_{\min }$ will increase with $\tilde{\omega}$ (see figure 4(a)). Here and below we use the reduced values of the time $\tilde{T}_{\mathrm{sw}}=T_{\mathrm{sw}} \omega_{\mathrm{r}} / 2 \pi$. The switching process through the non-uniform mode is characterized by very large values of $\tilde{T}_{\mathrm{sw}}$ and high sensitivity to the precision of $\tilde{h}$. When $\tilde{h} \rightarrow \tilde{h}_{\text {min }}, \tilde{T}_{\mathrm{sw}} \rightarrow \infty$, which is confirmed by the fact (see figure $4(\mathrm{a})$ ) that the dimensionless switching time $\tilde{T}_{\mathrm{Sw}}$ is determined only by the dimensionless waiting time $\tilde{T}_{\mathrm{w}}\left(\tilde{T}_{\mathrm{w}}=\right.$ $\left.\tilde{T}_{\mathrm{w}} \omega_{\mathrm{r}} / 2 \pi\right)$. Here the waiting time $T_{\mathrm{w}}$ is the time during which $\vec{\mu}$ dynamics is observed, or the maximal value of parameter $t$ during the numerical solution of the equations (5). Since the establishment of the equilibrium time, in the case of nonuniform precession, is very sensitive to the field amplitude, the waiting time $\tilde{T}_{\mathrm{w}}$ is the main limiting parameter in the procedure to numerically obtain $\tilde{h}_{\min }$. As far as the value of $\tilde{T}_{\mathrm{sw}}$ is calculated using the numerical procedure to find $\tilde{h}_{\text {min }}$, there are several flat curves in figure 4 . The value of $\tilde{T}_{\mathrm{w}}$ includes the time required for relaxation to the ground state after switching. This fact explains why $\tilde{T}_{\mathrm{sw}}<\tilde{T}_{\mathrm{w}}$ in that figure.

As shown above, the action of the permanent magnetic field along the easy axis leads to a reduction of $\tilde{\omega}$ and 
a)

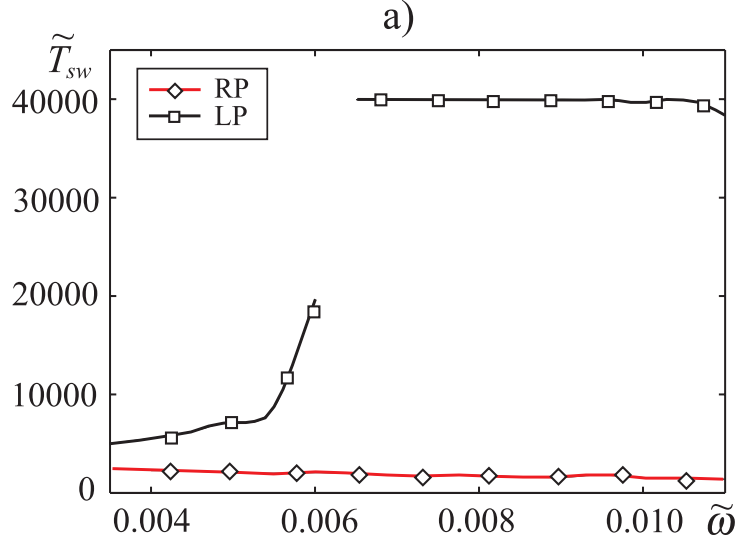

b)

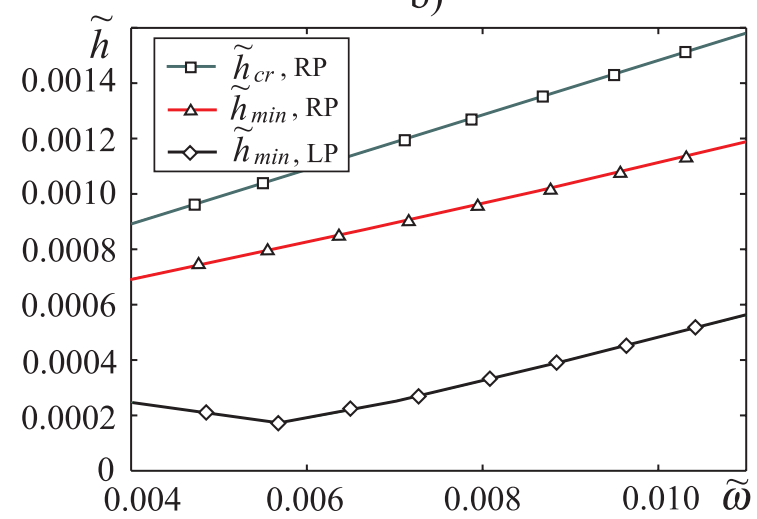

Figure 5. (a) Switching time, calculated in the resonant period for different waiting times for the case of $\tilde{H}=-0.99$; (b) values of minimal field's amplitudes for $\tilde{H}=-0.99$.

$\tilde{h}$ for the switching field. It follows from [28] however that switching under the action of such a field, or so-called damping switching, is characterized by a very large switching time. Therefore the goal of achieving a smaller rotating field amplitude and frequency by combining it with a permanent field $\tilde{H}$, will be offset by a delay of the reversal process (see figure $4(\mathrm{~b})$ ). The behavior of $\tilde{T}_{\mathrm{sw}}(\tilde{\omega})$ corresponding to $\tilde{h}_{\text {min }}$ is similar to the case of $\tilde{H}=0$. The difference is a sharp decrease of the switching times for large frequencies, as a result of overcoming the non-uniform mode during the transition process. The non-coincidence of the curves for large frequencies is explained by the high sensitivity of $\tilde{T}_{\mathrm{sw}}$ to the precision of $\tilde{h}$ and differences in waiting times.

A rotating field with right polarization can also produce switching in the presence of $\tilde{H}$, but the minimal field amplitude must be larger than for the left polarized field (see figure 2). This condition leads to a decrease of switching times in comparison with the left polarized case, see figure 5 . We want to emphasize however, that this situation is caused by different field amplitudes and, as we will show below, for equal amplitudes the left polarized field produces faster switching in comparison with the right polarized one.

When the condition $\tilde{h}>\tilde{h}_{\min }$ for the rotating field amplitude holds, it is reasonable to expect a significant decrease of the $\tilde{T}_{\mathrm{sw}}$. In fact, under certain circumstances,

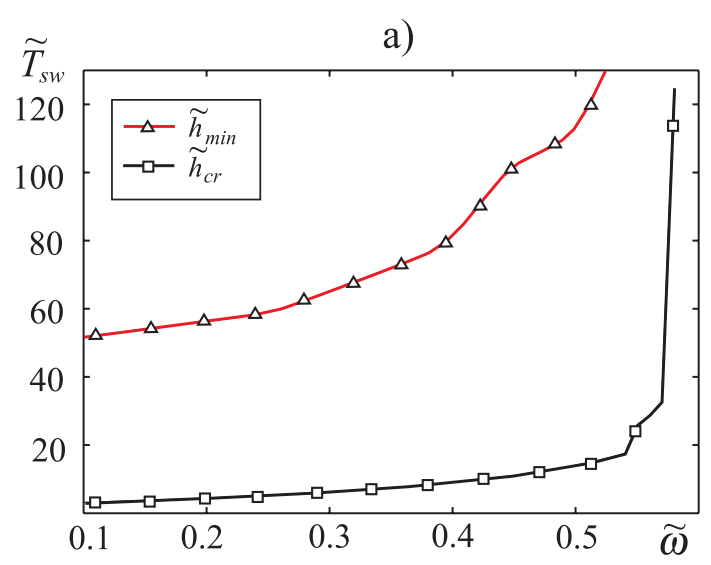

b)

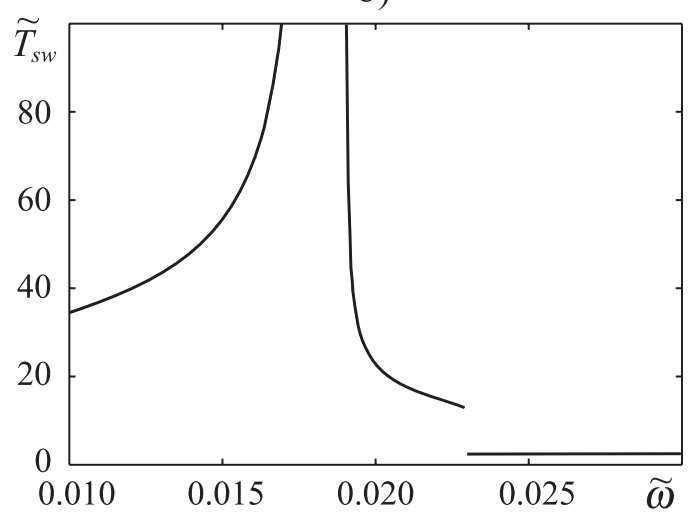

c)

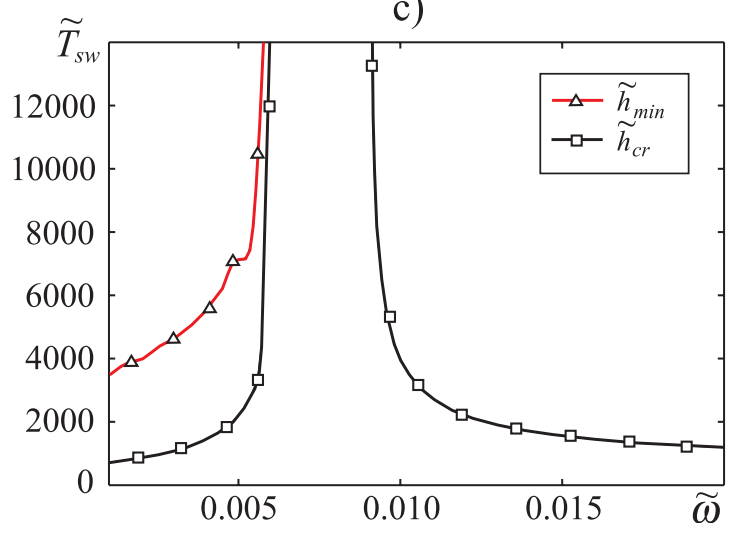

Figure 6. Switching time, calculated relative to the resonant period in the case of turning on the rotating field with amplitude $\tilde{h}=\tilde{h}_{\text {cr }}$.

(a) The case of $\tilde{H}=0$; (b) the case of $\tilde{H}=0$ and small frequencies; (c) the case of $\tilde{H}=-0.99$.

the switching time, contrary to expectations, can grow up to infinity (see figure 6). This can be explained as follows. The magnetic moment $\vec{\mu}$ of the nanoparticle under the action of an external field is in a double-well potential. The energy of the $\vec{\mu}$ has two minima: local with energy $W_{1}$ and global with energy $W_{\mathrm{g}}$. Let us assume that for $t=0$ the condition $\vec{\mu}=\vec{e}_{z}|\vec{\mu}|$ (or $\theta=0$ ) holds. The magnetization reversal process is the transition of $\vec{\mu}$ from the initial state to the global minimum, characterized by the angle $\theta_{\mathrm{g}}(>\pi / 2)$. During this process the magnetic moment can be captured by the local minimum, characterized by the angle $\theta_{1}(<\pi / 2)$. If the values of energies 
$W_{1}$ and $W_{\mathrm{g}}$ are very close, it takes a long time for $\vec{\mu}$ to leave the local minimum in favor of the global one. So, the switching time can reach huge values. We emphasize that this is a purely dynamical effect caused by turning on the external field and is not detected with quasistatic changes of the field amplitude. For $\tilde{H}=0$ this effect takes place for small $\tilde{\omega}$ and large $\tilde{h}$, whereas in the $\tilde{H}=-0.99$ case it is within the appropriate range of values $\tilde{\omega}$ and $\tilde{h}$. So, the combined action of rotating and permanent external fields is characterized by an extremely strong dependence of switching time on the field parameters.

\subsection{Switching time under pulsed field action}

In a real writing process the action of external fields has a restricted duration, and the shapes of the field pulse can be different. The switching behavior of $\vec{\mu}$ is defined by the length and shape of the pulse (see [37]). Since the pulse duration is determinative, we consider the simplest shape of the field pulse, i.e., the square one. For the best performance of media devices the pulse length has to be defined by the switching time. In turn, changing the length of the pulse can influence the switching time directly. During the transition processes, the sign of the projection of $\vec{\mu}$ on the $z$ axis can change several times. And if the external field will be turned off after the first crossing of the $x y$ plane by the vector $\vec{\mu}$, due to uniaxial anisotropy, the nanoparticle magnetic moment will stay in the down state. We designate the value of the rotating field amplitude, which corresponds to the minimal one in the case of a pulsed field, as $\tilde{h}_{\text {min }}^{\mathrm{p}}$. Since the condition $\tilde{h}_{\min }^{\mathrm{p}}<\tilde{h}_{\min }$ can hold for some frequencies (see figure $7(\mathrm{a})$ ), it is possible to reduce the minimal switching field amplitude in comparison with the case of the field turning on. As follows from figure 7(a), it is the normal situation for the nonuniform mode, when the amplitude of nutation can be rather large and the sign of the projection of $\vec{\mu}$ projection on the $z$ axis changes periodically. This provides the possibility of switching the nanoparticle magnetization despite the presence of the Q-mode. A similar idea was proposed in [27] within the approximation of a non-damped particle under the action of an external field $\vec{H}_{\perp}$ perpendicular to the easy axis of particle. On the other hand if the external rotating field is turned off, when the $\vec{\mu}$ projection on the $z$ axis is positive, the vector $\vec{\mu}$ will stay in the initial, or up, state. Thus, in order to ensure a sufficient level of reliability of writing in a media device, it is necessary to be very careful when using this effect for the writing process.

The dependence of the switching time on the reduced frequency for the case of the field turning off, when $\vec{\mu}$ crosses the $x y$ plane, is depicted in figure 7(b). There are four regions in the figure that are distinguished by the different by the different transition processes character. Here region 2 deserves special attention, because it corresponds to the minimal switching time, and also the minimal field amplitude.

It is expected that if the field amplitude is larger than the minimal one $\left(\tilde{h}>\tilde{h}_{\min }^{\mathrm{p}}\right)$, the duration of the pulse can be smaller. Figure 8 (a) shows the dependencies of the switching time on the field amplitude for a fixed frequency and pulse length $\tilde{T}_{\mathrm{p}}$. Here we can see that the switching of the nanoparticle magnetization occurs for pulse lengths of a)

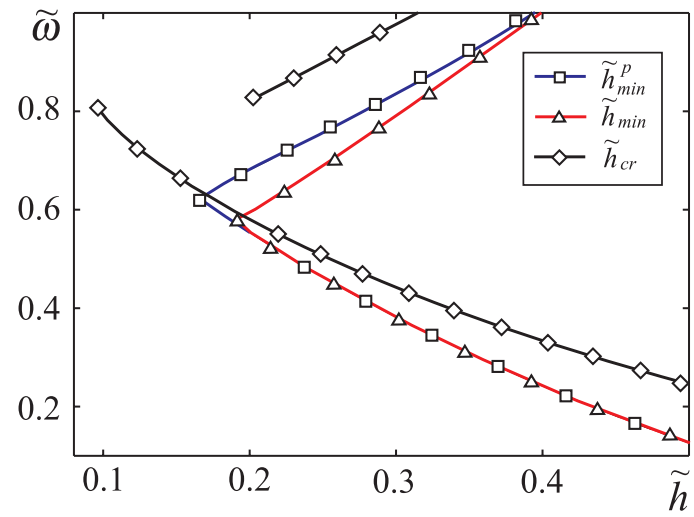

b)

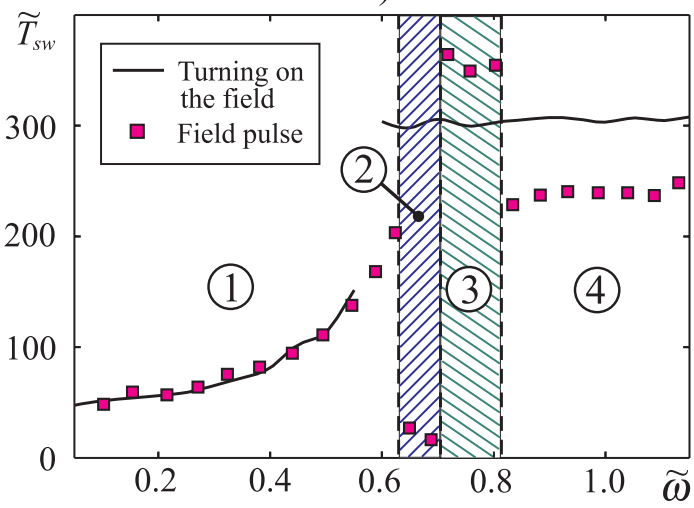

Figure 7. The case of turning off the field, when $\vec{\mu}$ cross the $x y$ plane. (a) Dependencies of the minimal field amplitude on frequency; (b) dependencies of the switching time on frequency: (1) the region, corresponding to the switching through the $\mathbf{P}$-mode, (2) the region, corresponding to the switching through the Q-mode with minimal switching times, (3) the region, corresponding to the switching through the $\mathbf{Q}$-mode with maximal switching times, (4) the region, corresponding to the switching through the $\mathbf{Q}$-mode with switching times weakly dependent on frequency.

several resonant periods only. From this figure the influence of the pulse length on $\tilde{T}_{\mathrm{sw}}$ is also clear. As expected, the switching time decreases with increasing field amplitude, but in addition breaks and gaps are observed. The presence of breaks is related to the crossing by the vector $\vec{\mu}$ of the $x y$ plane an odd number of times during the action of the field pulse. In turn, gaps take place, when $\vec{\mu}$ crosses the $x y$ plane an even number of times. In other words, even though in the equilibrium state, corresponding to the defined field amplitude $\tilde{h}$, the projection of magnetic moment $\vec{\mu}$ on $z$ axis is positive, during the establishment of equilibrium due to oscillations, the projection of the magnetic moment $\vec{\mu}$ on the $z$ axis can become negative. If we turn off the field when the projection is negative than the switching is completed. The reverse situation is also possible.

The same dependencies for the case of $\tilde{H}=-0.99$ are depicted in figure $8($ b). Here field $\tilde{H}$ was kept constant in time during the simulation. As expected from the previous results, the switching time in this case is larger by two orders of magnitude than for $\tilde{H}=0$. The next feature of the switching process is the inequality $\tilde{T}_{\mathrm{sw}}<\tilde{T}_{\mathrm{p}}$ for small $\tilde{h}$. This means 
a)

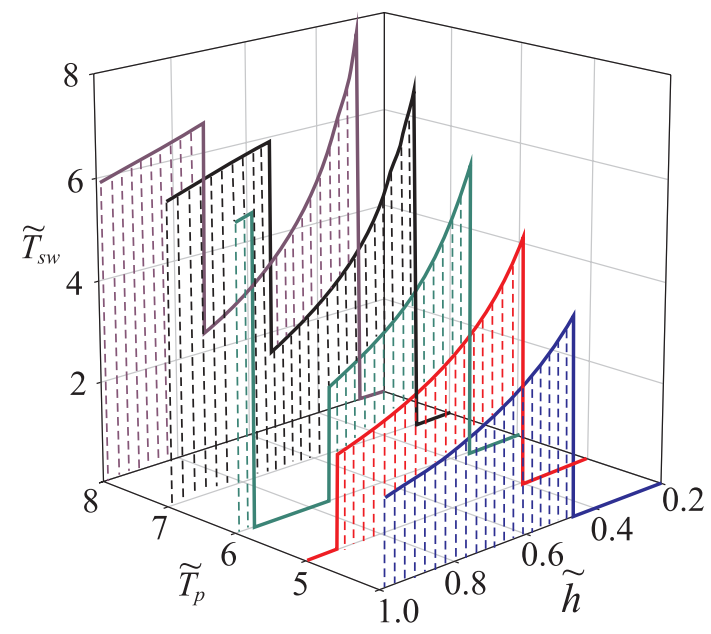

b)

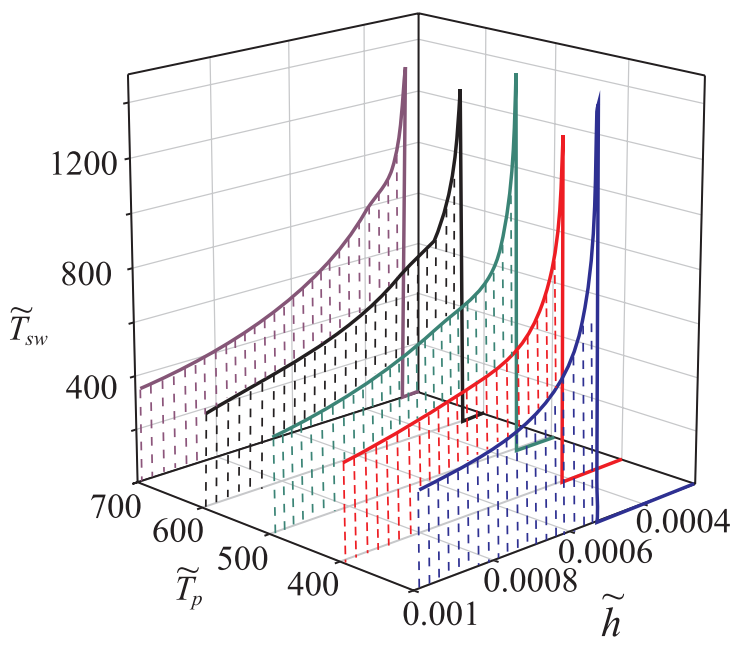

Figure 8. Switching time for different pulse lengths and amplitudes. (a) The case of $\tilde{H}=0$; (b) the case of $\tilde{H}=-0.99$.

that the rotating field acts as a small perturbation, that moves $\vec{\mu}$ from the equilibrium state (up state), and then the assistant field $\tilde{H}$ leads $\vec{\mu}$ to another equilibrium state (down state). Increasing $\tilde{T}_{\mathrm{p}}$ leads only to decreasing minimal amplitude $\tilde{h}$ of the circularly polarized switching field. The absence of breaks and gaps, as in the previous case, is explained by a single crossing of the $x y$ plane by $\vec{\mu}$ during the reversal process.

The advantages of using a circularly polarized field over a permanent one $\vec{H}_{\perp}$ perpendicular to the easy axis of the particle were revealed for the case of changes of the field amplitude in a quasistatic fashion (see figure 2(b)). It was shown there, that in the presence of a permanent field directed along the easy axis, rotating in a counterclockwise direction produces switching of the nanoparticle magnetization for smaller amplitudes in comparison with the field rotating in a clockwise direction. The field $\vec{H}_{\perp}$ corresponds to the rotating one with zero frequency and, as follows from figure 2(b), such a permanent switching field has a larger magnitude than the left polarized one. In figure 9 the results of numerical simulations for pulsed fields

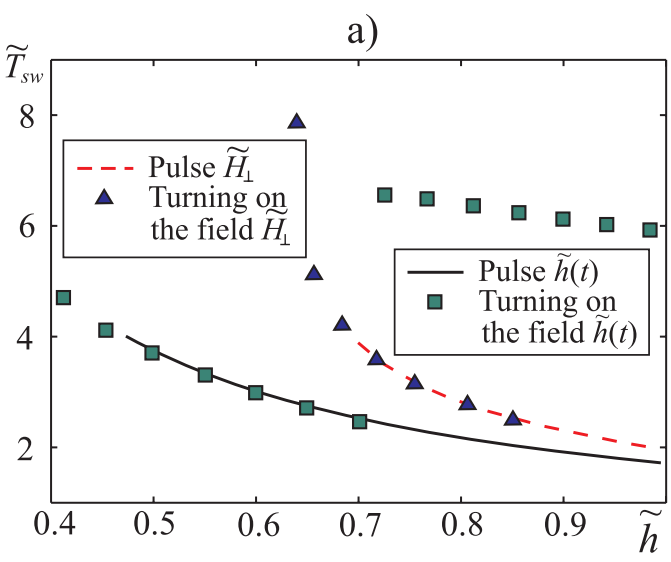

b)

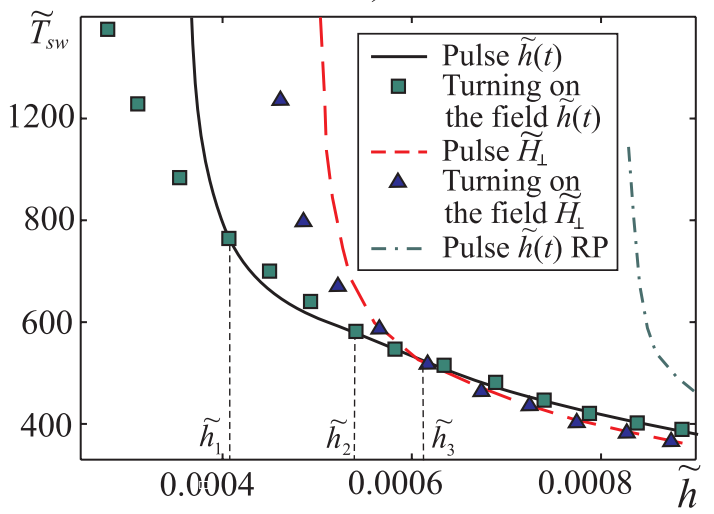

Figure 9. Switching time for pulsed action of the rotating field and permanent one, perpendicular to the easy axis. (a) The case of $\tilde{H}=0, \tilde{\omega}=0.6, \tilde{T}_{\mathrm{p}}=4$; (b) the case of $\tilde{H}=-0.99, \tilde{\omega}=0.006$, $\tilde{T}_{\mathrm{p}}=500$.

$\vec{H}_{\perp}$ and $\vec{h}(t)$ are depicted. Here the frequency and the pulse length $\tilde{T}_{\mathrm{p}}$ are fixed, and the switching time is determined as a function of the field magnitude. When the condition $\tilde{H}=0$ holds, the pulsed action of the rotating field provides smaller switching times for large $\tilde{h}$ in comparison with the case of a simple turning on of the field. It occurs because the end of the pulse does not let the vector $\vec{\mu}$ cross the $x y$ plane a second time. Also, following from the presented results we find considerably better switching parameters of the circularly polarized field pulse in comparison with a pulse of the permanent field $\tilde{H}_{\perp}$.

The case of the pulsed action of the field $\vec{h}(t)$, assisted by the permanent field $\tilde{H}=-0.99$, is more complicated (see figure 9 (b)). Here the advantages of the pulsed action exist only for a fixed interval of the field amplitudes $\tilde{h}_{1}<\tilde{h}<$ $\tilde{h}_{2}$ (see figure $9(\mathrm{~b})$ ). Finally, a circularly polarized field is characterized by smaller switching times and magnitudes in comparison with the action of the permanent field $\tilde{H}_{\perp}$, when the condition $\tilde{h} \leqslant \tilde{h}_{3}$ holds. If $\tilde{h}>\tilde{h}_{3}$, the switching time for the rotating field exceeds $\tilde{T}_{\mathrm{sw}}$ for the field $\tilde{H}_{\perp}$, but the difference between them is not too large. For comparison, in figure 9(b) the dependencies for right polarized field are depicted. As we can see, its switching characteristics are worse. So, as a result, a circularly polarized field with left polarization is more appropriative for magnetization switching. 


\section{Conclusions}

The magnetization switching of a ferromagnetic uniaxial nanoparticle under the combined action of a circularly polarized field and a static one directed along the easy axis was studied in a coherent rotation approximation. Within this framework it was shown numerically, that the switching or generation of the non-uniform mode occurs when the precession of the nanoparticle magnetic moment becomes unstable. The conditions for the existence of uniform and non-uniform precession of the nanoparticle magnetic moment were revealed. Switching through the non-uniform mode of precession was observed, but the switching time in this case can be very large, up to infinity. It was shown that the application of an external permanent field along the easy axis of the particle leads to a reduction of the values of the rotating field frequency and amplitude for the switching, but also increases the switching time. In addition, it was demonstrated that the assistance of such a permanent field permits the reversal of the nanoparticle magnetization by a field polarized in the direction opposite to the natural precession. In this case however switching parameters are poor in comparison with the action of a switching field rotating in the natural precession direction.

It was confirmed that transition processes, which occur during the turning on of the rotating field, can reduce the switching field amplitude in comparison with that predicted analytically. For the case of an assistant permanent field applied along the easy axis, such transition processes can overcome the non-uniform mode for fixed values of frequencies. Also the switching time under the action of a pulsed field was studied. It was shown that the pulse field can decrease the switching time and field amplitude in comparison with the permanent external field. Depending on pulse duration, the nanoparticle magnetic moment can cross the $x y$ plane an even or odd number of times, but the switching effect takes place for the latter case only. Therefore there are rigid requirements of the pulse length to provide a satisfactory level of reliability in the writing process.

Finally, it was shown, that the amplitude of the field, rotating in the direction of natural precession, and switching time, are smaller in comparison with the case of a permanent field perpendicular to the easy axis of the particle. This confirms that the field circularly polarized in the plane perpendicular to the easy axis is more appropriative for the magnetization switching than the permanent one.

\section{Acknowledgments}

Authors acknowledge the support of the EU through NANOSPIN contract No NMP4-CT-2004-013545. We are grateful to S I Denisov for a critical reading of the manuscript and his valuable comments.

\section{References}

[1] Berkowitz A 1992 Phys. Rev. Lett. 683745

[2] Xiao J 1992 Phys. Rev. Lett. 683749

[3] Gunther L and Barbara B (ed) 1995 Quantum Tunneling of Magnetization-QTM'94 (Dordrecht: Kluwer)

[4] Chudnovsky E and Tejada J 1998 Macroscopic Quantum Tunneling of the Magnetic Moment (Cambridge: Cambridge University Press)

[5] Nogués J 2005 Phys. Rep. 42265

[6] Fiorani D (ed) 2005 Surface Effects in Magnetic Nanoparticles (Berlin: Springer)

[7] Plumer M, Van Ek J and Weller D (ed) 2001 The Physics of Ultra-High-Density Magnetic Recording (Berlin: Springer)

[8] Albrecht M, Rettner C and Moser A 2002 Appl. Phys. Lett. 812875

[9] Chien C L, Frank Q Z and Jian-Gang Z 2007 Phys. Today 6040

[10] Black C T et al 2005 United States Patent Application 20050152073

[11] Stoner E and Wohlfarth E 1948 Phil. Trans. Roy. Soc. Lon. A 240599

Stoner E and Wohlfarth E 1991 IEEE Trans. Magn. 273475 (Reprinted)

[12] Wernsdorfer W et al 1997 Phys. Rev. Lett. 781791

[13] Brown W 1963 Phys. Rev. 1301677

[14] Dormann J L, Bessasis L and Fiorani D 1998 J. Phys. C: Solid State Phys. 212015

[15] Mørup S and Tronc E 1994 Phys. Rev. Lett. 723278

[16] Lottis D K, White R M and Dan Dahlberg E 1991 Phys. Rev. Lett. 67362

[17] Denisov S I and Trohidou K N 2001 Phys. Rev. B 64184433

[18] Kanai Y and Charap S H 1991 IEEE Trans. Magn. 274972

[19] Suran G et al 2000 Europhys. Lett. 41671

[20] Nowak U, Chantrell R W and Kennedy E C 2000 Phys. Rev. Lett. 84163

[21] Denisov S I, Lyutyy T V and Trohidou K N 2003 Phys. Rev. B 67014411

[22] van Lierop J and Ryan D H 2001 Phys. Rev. B 63064406

[23] Jonsson T, Nordblad P and Svedlindh P 1998 Phys. Rev. B 57497

[24] Dormann J L et al 1998 J. Magn. Magn. Mater. 187 L139

[25] Stoner E and Wohlfarth E 1948 Phil. Trans. R. Soc. 240599

[26] Stamp R and Hillebrands B 1999 Appl. Phys. Lett. 751143

[27] Serpico C, Mayergoyz I and Bertotti G 2003 J. Appl. Phys. 936909

[28] Bertotti G, Mayergoyz G, Serpico C and Dimian M 2003 J. Appl. Phys. 936811

[29] Khapikov A 1992 JETP Lett. 55352

[30] Thirion C, Wernsdorfer W and Mailly D 2003 Nat. Mater. 2524

[31] Sun Z and Wang X 2006 Phys. Rev. B 74132401

[32] Denisov S I, Lyutyy T V, Hänggi P and Trohidou K N 2006 Phys. Rev. B 74104406

[33] Denisov S I, Lyutyy T V, Hänggi P and Trohidou K N 2006 Phys. Rev. Lett. 97227202

[34] Bertotti G, Serpico C and Mayergoyz I 2001 Phys. Rev. Lett. 86724

[35] Sun Z and Wang X 2006 Phys. Rev. Lett. 97077205

[36] Landau L and Lifshitz E 1935 Phys. Z. Sowjetunion. 8153

[37] Bauer M, Fassbender J, Hillebrands B and Stamp R 2000 Phys. Rev. B 613410 